Proc. 19th Winter Workshop on

Nuclear Dynamics (2003) 000-000

\begin{tabular}{l}
\hline 19th Winter Workshop \\
on Nuclear Dynamics \\
Breckenridge, Colorado, USA \\
February 8-15, 2003 \\
\hline \hline
\end{tabular}

\title{
Do Ordinary Nuclei Contain Exotic States of Matter?
}

\author{
John Arrington ${ }^{1}$ \\ 1 Argonne National Laboratory, \\ Argonne, IL, 60439, USA
}

\begin{abstract}
The strongly repulsive core of the short-range nucleon-nucleon interaction leads to the existence of high-momentum nucleons in nuclei. Inclusive electron scattering can be used to probe these high-momentum nucleons and study the nature of the corresponding short-range correlations in nuclei. With recent data from Jefferson Lab we have begun to map out the strength of two-nucleon correlations in nuclei, while upcoming experiments should allow us to isolate the presence of multi-nucleon correlations. In addition to their importance in describing nuclear structure, these configurations of correlated nucleons represent high density 'droplets' of hadronic matter. As the density of hadronic matter increases there should be a weakening of quark confinement, similar to the onset of deconfinement expected at extremely high temperatures. While there have been hints of non-hadronic structure in nuclei, future measurements will allow us to directly probe the quark distributions of high density configurations in nuclei. A modified quark structure in these closely packed nucleons would provide a clear signature of exotic components to the structure of nuclei.
\end{abstract}

Keywords: keywords here

PACS: $12.38 . \mathrm{Mh}, 24.85 .+\mathrm{p}, 25.30 . \mathrm{Fj}$

\section{Inclusive Scattering from Nuclei at $x>1$}

Inclusive scattering from nuclei at $x>1$ has provided a great deal of information about the high momentum components of the nuclear wave function. Measurements at large momentum transfer, $q$, and small energy transfer, $\nu$, are dominated by quasielastic scattering from single nucleons. This process is sensitive to the elastic electron-nucleon cross section and the distribution of nucleons within the nucleus. For a stationary nucleon, elastic scattering corresponds to $x_{\text {Bjorken }}=Q^{2} / 2 m_{N} \nu=$ 1 , where $m_{N}$ is the mass of the nucleon and $Q^{2}=q^{2}-\nu^{2}$. Nucleons with a large initial momentum parallel (anti-parallel) to the exchange photon occur at $x>1$ 
$(x<1)$. By selecting low energy transfers, corresponding to $x>1$, there is little background from inelastic processes, and the measurements are sensitive to the distribution of nucleons with large momenta.

These high momentum components come from short-range correlations (SRCs) in the nucleus. These correlations are an important part of nuclear structure and are related to the short-range, strongly repulsive core of the N-N interaction. Comparisons of inclusive scattering from heavy nuclei and deuterium provided one of the first experimental signatures of short-range range correlations in nuclei [ 1] 22. Above $x \approx 1.5$, the cross section behavior was identical for all nuclei measured, yielding a plateau in the $A / D$ ratio and indicating that the same two-nucleon correlations that generate the high-momentum nucleons in the deuteron are also the dominant source of high-momentum nucleons in heavy nuclei. Subsequently, shortrange correlations in nuclei have been probed in a variety of reactions including $\mathrm{A}(\mathrm{p}, 2 \mathrm{p}), \mathrm{A}\left(\mathrm{e}, \mathrm{e}^{\prime} \mathrm{p}\right), \mathrm{A}\left(\mathrm{e}, \mathrm{e}^{\prime} \mathrm{NN}\right)$.

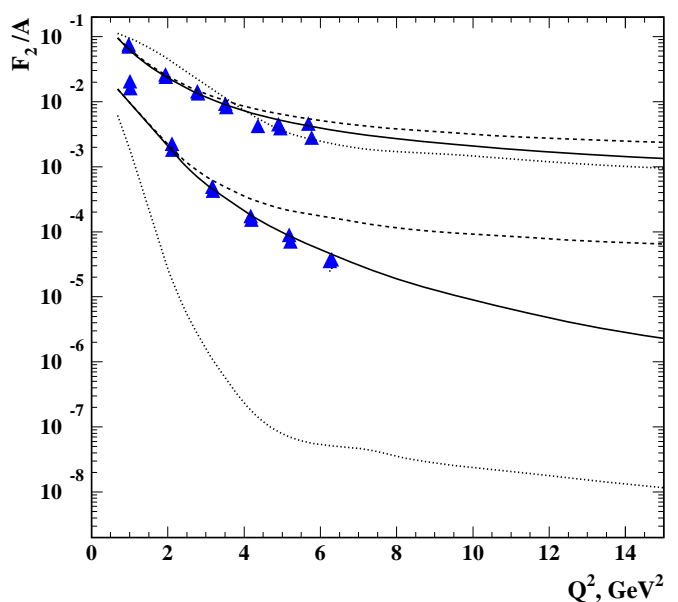

Fig. 1. Structure function per nucleon for iron [3] from JLab E89-008 compared to calculations without correlations (dotted lines), including two-nucleon SRCs (solid lines), and multi-nucleon SRCs (dashed lines). The upper set of data and calculations is for $x=1$, while the lower are for $x=1.5$. The $6 \mathrm{GeV}$ extension will extend coverage to $Q^{2}=8(10) \mathrm{GeV}^{2}$ at $x=1(1.5)$.

Completed and planned measurements [3, 4] at Jefferson Lab up to $6 \mathrm{GeV}$ will map out the strength of two-nucleon short-range correlations in nuclei. These measurements will also provide a first look at high- $Q^{2}$ cross sections for $2.5 \leq$ $x<3$, where the cross section is most sensitive to the presence of multi-nucleon correlations. Figure 1 shows the structure function per nucleon for iron compared to a mean-field calculation, a calculation including two-nucleon SRCs [ 2], and one including multi-nucleon SRCs [ [5]. The $4 \mathrm{GeV}$ data are consistent with twonucleon SRCs, while future measurements at $6 \mathrm{GeV}$ [4] will significantly increase 
the sensitivity to multi-nucleon correlations, extending the $Q^{2}$ range at $x=1.5$ to more than $10 \mathrm{GeV}^{2}$. Even higher beam energies will dramatically increase both the $x$ and $Q^{2}$ range of the data. With the increased $x$ coverage possible with an 11 $\mathrm{GeV}$ beam at Jefferson Lab, these studies can be extended to isolate and map out the strength of multi-nucleon correlations.

In addition to improving the sensitivity to multi-nucleon correlations, higher energies will also provide a new way to study two-nucleon correlations by allowing us to probe the quark structure of two-nucleon correlations. A measurement in the deep inelastic scattering regime at $x>1$ would allow us to measure for the first time the distributions of superfast quarks in nuclei, where the struck quark carries more momentum than an entire nucleon.

There is little data available for structure functions of nuclei at $x>1$. Measurements of muon scattering from iron [ [6] have only upper limits above $x=1.1$, and show a rapid falloff in the structure function near $x=1\left(F_{2} \propto e^{-16.5 x}\right)$. Measurements of neutrino scattering from carbon [ 7] also have a limited $x$ range $(x<1.2)$, but indicate significantly more strength at large $x\left(F_{2} \propto e^{-8.3 x}\right)$. With the increase in $Q^{2}$ possible with the JLab energy upgrade, high precision measurements of the structure function over a wide range in $x$, allowing us to cleanly map out the distribution of superfast quarks. At the $Q^{2}$ values achievable with the JLab upgrade, the inelastic cross section dominates even for $x>1$, and we should be able to extract the structure function in the scaling regime up to $x \approx 1.4$.

\section{High-density Configurations}

These high- $x$ quark distributions are intrinsically related to the quark structure of the two-nucleon correlations. Fig. 1 demonstrates that for large $x$ and $Q^{2}$, the scattering is dominated by scattering from these short-range configurations. These quark distributions probe the internal structure of high-density configurations, where nucleons have significant overlap. The EMC effect demonstrates that the quark structure of a nucleus is more than just a convolution of the quark structure of its nucleons. If this comes from density-dependent modifications to nucleon structure, then a similar but much larger effect should be seen when one examines the quark distributions at large $x$, where the strength comes almost entirely from nucleon pairs that are nearly overlapping [ 8$]$.

Such a modification of hadron structure is expected at high densities. Figure 2 shows the phase diagram for nuclear matter. At high densities, just as at high temperature, there is a phase transition from hadronic matter to quark matter. While ordinary nuclear densities are well below this transition, the densities achieved in overlapping nucleons may be high enough to see the effect of this phase transition. Thus, the EMC effect may not arise from the increase in average nuclear density but instead a be result of large changes in the structure of the high-density components arising from short-range correlations.

Figure 3 illustrates the overlap of nucleons separated by $1.7 \mathrm{fm}$, the average 


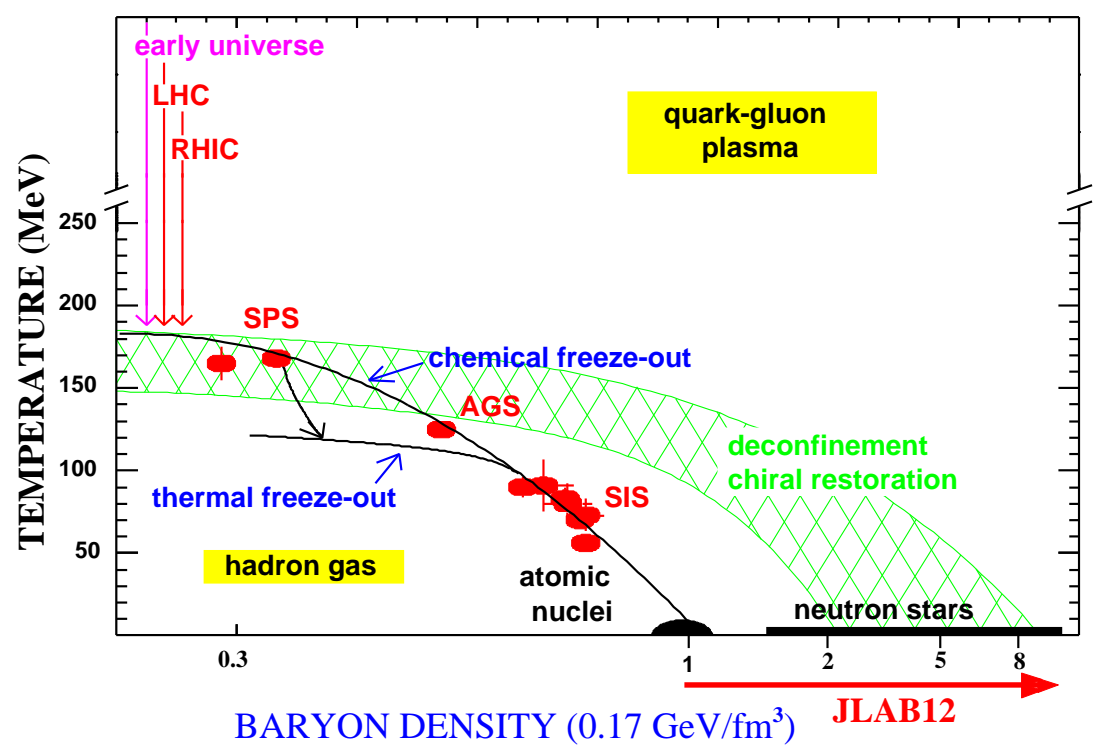

Fig. 2. Phase diagram for nuclear matter.

separation in nuclear matter, and $0.6 \mathrm{fm}$. The short-range repulsive core of the N-N interaction becomes very large below $\approx 0.4 \mathrm{fm}$, so the separation of the nucleons in the correlated pair should be larger than this distance. At $0.6 \mathrm{fm}$, the peak densities are several times normal nuclear matter densities.

At these densities, there may be a significant change in the structure of the nucleon, which will manifest itself in a modified quark distributions. Figure 4 shows the quark distribution for a deuteron made of just two nucleons (dashed line), and with a $5 \%$ contribution from a 6 -quark bag (solid line). For $x<1$, there is almost no difference in the quark distributions, but for $x>1$ the small 6-quark component dominates the quark distribution. While this is just one model for nuclear effects at high density, a significant increase in the distribution of superfast quarks is a fairly general signature of such modifications. In a nucleon at rest the quarks share its momentum, and thus cannot have a momentum fraction, $x$, larger than one. In nuclei, quarks can gain additional momentum from the interaction of the nucleons. Even taking into account the high-momentum tails of the nucleon distributions coming from SRCs, the quark distribution falls off extremely rapidly for $x>1$. However, if the nucleons in the SRCs have significant overlap, the quarks can directly share their momentum, allowing for a much greater probability of finding a single quark with a very large momentum fraction. Such an observation would be a clear signature of deviations from the purely hadronic picture of nuclei. 

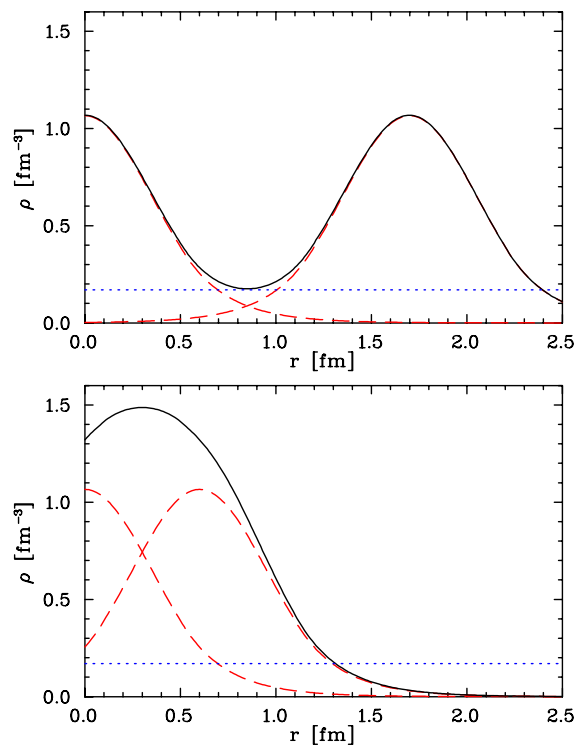

Fig. 3. One dimensional density profile for two nucleons separated by $1.7 \mathrm{fm}$ (top) and $0.6 \mathrm{fm}$ (bottom).

\section{Conclusions}

Measurements of inclusive scattering from nuclei at $x>1$ probe the high-momentum components of the nuclear wavefunction. These high momentum nucleons arise from the short-range interaction of nucleon pairs. At moderate energy scales, where the scattering is dominated by quasielastic scattering from individual nucleons, these nucleon pairs can be kinematically isolated in inclusive reactions. High energy measurements will allow us to probe the quark structure of these short-range correlations, and look for modification to the underlying quark structure of hadrons in regions where the instantaneous densities are several times the density of normal nuclear matter. The structure of nucleons and the distribution of high-momentum quarks may be substantially altered in this region, where significant overlap of nucleons should allow direct interaction between quarks in different nucleons. Studies of these high-density configurations will allow us to determine if such modifications of nucleon structure are responsible for the EMC effect, and will help us understand the quark-gluon phase transition at high density. This complements RHIC studies of this same transition at high temperature, while at the same time providing us information on the structure of matter at extremely high densities. Probing these high density components in nuclei is the only way to directly study high density nuclear matter, and what we learn here will be important in understanding neutron stars and other compact astronomical objects. 

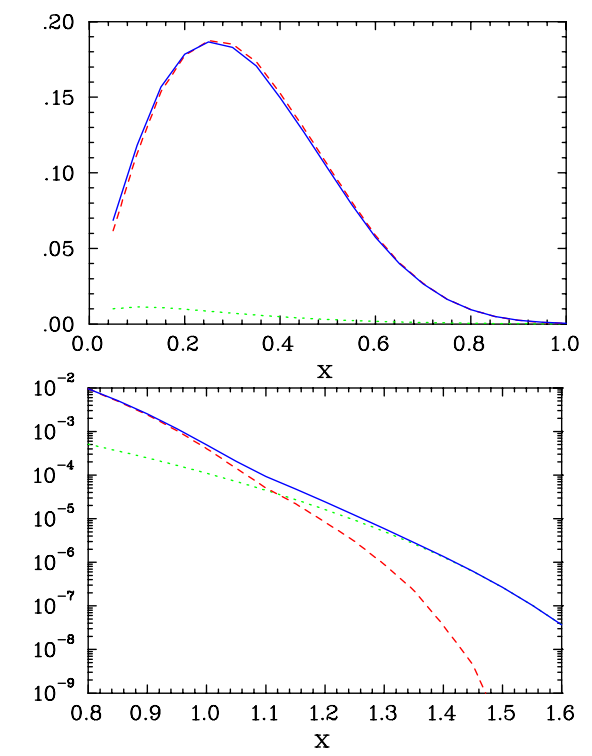

Fig. 4. Quark distributions for deuteron assuming only 2-nucleon components (dashed line), and assuming a 5\% contribution from a 6-quark bag (solid line). The dotted line shows the $5 \%$ 6-quark component [9].

\section{Acknowledgments}

This work is supported by the U.S. Department of Energy, Nuclear Physics Division, under contract W-31-109-ENG-38.

\section{References}

1. D. B. Day, Nucl. Phys. A478, 397 (1988)

2. L. L. Frankfurt, M. I. Strikman, D. B. Day, and M. Sargsian, Phys. Rev. C 48, 2451 (1993).

3. J. Arrington et al. Phys. Rev. Lett. 82, 2056 (1999)

4. Jefferson Lab experiment E02-019: J. Arrington, D. B. Day, B. W. Filippone, A. Lung spokespersons.

5. L. L. Frankfurt and M. I. Strikman, Phys. Lett. B94, 216 (1980).

6. Benvenuti et al. Zeit. Phys. C63, 29 (1994).

7. M. Vakili et al. Phys. Rev. D61, 052003 (2000).

8. M. M. Sargsian, et al., J. Phys. G29: R1 (2003).

9. P. Mulders and A. W. Thomas, Phys. Rev. Lett. 52, 1199 (1984). 